KUNS-1298

$\mathrm{HE}(\mathrm{TH}) 94 / 14$

hep-ph/9411201

\title{
Possible Candidates for SUSY SO(10) Model with an Intermediate Scale
}

\author{
Masako Bando * \\ Physics Division, Aichi University, Aichi 470-02, Japan \\ Joe Sato ${ }^{\dagger}$ and Tomohiko Takahashi ${ }^{\ddagger}$ \\ Department of Physics, Kyoto University, Kyoto 606-01, Japan
}

July 26, 2021

\begin{abstract}
We study the possibility of an intermediate scale existing in supersymmetric $S O(10)$ grand unified theories: The intermediate scale is demanded to be around $10^{12} \mathrm{GeV}$ so that neutrinos can obtain masses suitable for explaining the experimental data on the deficit of solar neutrino with Mikheev-SmirnovWolfenstein solution and the existence of hot dark matter. We show that any Pati-Salam type intermediate symmetries are excluded by requiring reasonable conditions and only $S U(2)_{L} \times S U(2)_{R} \times S U(3)_{C} \times U(1)_{B-L}$ is likely to be realized as an intermediate symmetry.
\end{abstract}

${ }^{*}$ e-mail address: mband@jpnyitp.bitnet

${ }^{\dagger}$ e-mail address: joe@gauge.scphys.kyoto-u.ac.jp

${ }_{\ddagger}^{\ddagger}$ e-mail address: tomo@gauge.scphys.kyoto-u.ac.jp 
In constructing a realistic unified theory of matters and fields, it is inevitable to answer a question about neutrino masses. There seems to exist experiments indicating the neutrino masses and their mixing [1]: some experiments show a deficit of the solar neutrino, which may be explained by Mikheev-Smirnov-Wolfenstein (MSW) solution [2]. For example, according to one of MSW solutions, the mass of muon neutrino seems to be $m_{\nu_{\mu}} \simeq 10^{-3} \mathrm{GeV}$. Those small masses may be explained by the see-saw mechanism [3]: muon neutrino can acquire such a small mass if Majorana mass of right-handed muon neutrino is about $10^{12} \mathrm{GeV}$. Furthermore if all Majorana masses of right-handed neutrinos are of about $10^{12} \mathrm{GeV}$, the see-saw mechanism leads to the mass of tau neutrino $m_{\nu_{\tau}} \simeq 10 \mathrm{eV}$, which is desirable for the interpretation that tau neutrino may be hot dark matter. In the framework of $S O(10)$ Grand Unified Theory (GUT) [5], we can naturally incorporate right-handed neutrinos into ordinary fermions.

On the other hand it is well known that in Minimal Supersymmetric Standard Model (MSSM) the present experimental values of gauge couplings are successfully unified at a unification scale $M_{U} \simeq 10^{16} \mathrm{GeV}$ [6].

Then how can the right-handed neutrinos acquire masses of about $10^{12} \mathrm{GeV}$ when we have no scale other than $M_{U}$ ? There are several possibilities for the right-handed neutrinos to obtain masses of the intermediate scale, $M_{R} \simeq 10^{12} \mathrm{GeV}$. First, radiative correction of GUT scale physics, what we call Witten mechanism [4], can induce $M_{R}$. In a supersymmetric model, however, this mechanism cannot work because the nonrenormalization theorem [7] does protect inducement of terms via radiative corrections which are not contained in an original Lagrangian. The second possibility is that the Yukawa coupling of right-handed Majorana neutrino is so small that the mass may be the intermediate scale even if it originates in GUT scale. Thirdly singlet Higgs particles develop the vacuum expectation value at the intermediate scale mass to supply the mass of $M_{R}$ to $\nu_{R}$. In unrenormalizable models such as supergravity those latter two possibilities may be realized.

Our point of view is, however, that it is more natural to consider that one energy scale corresponds to a dynamical phenomenon, for instance a symmetry breaking. Thus we are led to another possibility that a certain group breaks down to the standard group at the intermediate scale at which right-handed neutrinos gain masses. This idea is consistent with the survival hypothesis. There are some papers which indicate that intermediate groups can enter in breaking chains of groups from GUT to Standard Model (SM) consistently with the analyses of MSSM such as coupling unification [8], but models presented in these papers do not involve Higgs contents which are able to provide masses to right- 
handed neutrinos. We would like to explain right-handed neutrino masses according to a symmetry breaking.

This paper is devoted to an investigation of a possibility that right-handed neutrinos acquire masses of order $10^{12} \mathrm{GeV}$ through a symmetry breaking within SUSY SO(10) GUT with one intermediate scale below which MSSM is realized. First we show assumptions about models and constraints on our analysis. Next we explain our analysis. Then we show results. Finally we give a summary.

First of all we show the breaking patterns allowed in our scenario,

$$
\begin{aligned}
& \text { I. } S O(10) \longrightarrow S U(2)_{L} \times S U(2)_{R} \times S U(4)_{P S}\left(G_{224}\right) \longrightarrow \text { MSSM, } \\
& \text { II. } S O(10) \longrightarrow S U(2)_{L} \times S U(2)_{R} \times S U(3)_{C} \times U(1)_{B-L}\left(G_{2231}\right) \longrightarrow \text { MSSM, } \\
& \text { III. } S O(10) \longrightarrow S U(2)_{L} \times U(1)_{R} \times S U(4)_{P S}\left(G_{214}\right) \longrightarrow \text { MSSM. }
\end{aligned}
$$

The first assumption is that there is at least one Higgs multiplet which is able to supply the intermediate scale mass to right-handed neutrinos besides ordinary matter (ordinary fermions, right-handed neutrinos and two Higgs doublets).

The second is that candidates for the matter contents in the intermediate region are multiplets included in representations 10, 16, 45, 54, 120, 126 and 210 of $S O(10)$.1

Phenomenologically we impose further constraints to our models.

1. The unified scale $M_{U}$ is larger than $10^{16} \mathrm{GeV}$. This is necessary for suppression of proton decay [9].

2. The intermediate scale is taken at $10^{10}, 10^{11}, 10^{12}, 10^{13}$ or $10^{14} \mathrm{GeV}$ because of right-handed neutrino masses. The reason why an intermediate scale is an input will be made clear after eq.(4).

3. The unified gauge coupling $\alpha_{U}\left(M_{U}\right)$ satisfies $\alpha_{U}^{-1}\left(M_{U}\right) \geq 5$. This constraint is required for a perturbative region to expand to GUT scale.

4. Any colored Higgs is not contained in the intermediate physics. This is needed also for suppression of proton decay [9].

Under these conditions, we specify combinations of matters mentioned above which realize the unification of gauge couplings to achieve GUT with a simple group.

\footnotetext{
${ }^{1}$ This is just an assumption. In general, however, models mentioned above are difficult to be accompanied by a representation which is contained in a higher representation of $S O(10)$ only. See a statement below eq. (9) and the summary for an example.
} 
Next we show the condition on the beta functions in the intermediate scale in order to achieve the unification of gauge couplings. In the following we made an analysis based on RGE up to one loop. The conditions of the unification are described by

$$
\begin{aligned}
\alpha_{Y}^{-1}\left(M_{S}\right) & =\alpha_{U}^{-1}\left(M_{U}\right)+\frac{1}{2 \pi} b_{Y} R+\frac{1}{2 \pi} b_{Y}^{\prime}(U-R), \\
\alpha_{L}^{-1}\left(M_{S}\right) & =\alpha_{U}^{-1}\left(M_{U}\right)+\frac{1}{2 \pi} b_{L} R+\frac{1}{2 \pi} b_{L}^{\prime}(U-R), \\
\alpha_{C}^{-1}\left(M_{S}\right) & =\alpha_{U}^{-1}\left(M_{U}\right)+\frac{1}{2 \pi} b_{C} R+\frac{1}{2 \pi} b_{C}^{\prime}(U-R),
\end{aligned}
$$

$b_{i}(i=Y, L, C)$ 's with dash and without dash denote the beta function in the lower scale and higher scale than the intermediate scale $M_{R}$, respectively. $\mathrm{R}$ and $\mathrm{U}$ are defined by

$$
R=\ln \frac{M_{R}}{M_{S}}, \quad U=\ln \frac{M_{U}}{M_{S}} .
$$

These equations lead to the relation which $R$ and $U$ must satisfy,

$$
\begin{aligned}
& \left(b_{Y}-b_{L}\right) R+\left(b_{Y}^{\prime}-b_{L}^{\prime}\right)(U-R)=2 \pi\left(\alpha_{Y}^{-1}\left(M_{S}\right)-\alpha_{L}^{-1}\left(M_{S}\right)\right), \\
& \left(b_{C}-b_{L}\right) R+\left(b_{C}^{\prime}-b_{L}^{\prime}\right)(U-R)=2 \pi\left(\alpha_{C}^{-1}\left(M_{S}\right)-\alpha_{L}^{-1}\left(M_{S}\right)\right) .
\end{aligned}
$$

Here we have assumed that in the lower scale MSSM is realized, so the equation (4) has always a solution $U=R \simeq 10^{16} \mathrm{GeV}$, which corresponds to the case that there is no intermediate scale physics. Therefore if there is a nontrivial intermediate scale $R$, the beta functions must satisfy the following condition,

$$
\left(b_{Y}-b_{L}\right)\left(b_{C}^{\prime}-b_{L}^{\prime}\right)-\left(b_{C}-b_{L}\right)\left(b_{Y}^{\prime}-b_{L}^{\prime}\right)=0 \text {. }
$$

Since the beta functions in MSSM are given by

$$
b_{Y}=\frac{33}{5}, \quad b_{L}=1, \quad b_{C}=-3,
$$

the beta functions between the intermediate scale and GUT scale must satisfy the equation,

$$
5 b_{Y}^{\prime}-12 b_{L}^{\prime}+7 b_{C}^{\prime}=0
$$

which we call "the unification condition". This is a sufficient condition on the gauge coupling unification under the assumption that MSSM is realized in the lower scale. When

\footnotetext{
${ }^{2}$ Though $b_{Y}^{\prime}=b_{L}^{\prime}=b_{C}^{\prime}$ satisfies the unification condition, in this case the condition that all couplings are unified is not fulfilled. Therefore this case is excluded.
} 
the equation (7) is satisfied, $\mathrm{R}$ becomes an arbitrary parameter. Therefore we introduce an intermediate scale.

We make an analysis as follows using the unification condition for the beta functions in addition to the above restrictions. Taking one combination of matter contents on the intermediate physics, we see whether the unification condition is fulfilled or not. If it is the case, we can calculate the unified scale $M_{U}$ and the gauge coupling $\alpha_{U}\left(M_{U}\right)$ at the unified scale using following equations,

$$
\begin{aligned}
M_{U} & =M_{R} \exp \left(2 \pi \frac{\alpha_{Y}^{-1}\left(M_{R}\right)-\alpha_{L}^{-1}\left(M_{R}\right)}{b_{Y}^{\prime}-b_{L}^{\prime}}\right) \\
\alpha_{U}\left(M_{U}\right) & =\left(\alpha_{L}^{-1}\left(M_{R}\right)-\frac{1}{2 \pi} b_{L}^{\prime}(U-R)\right)^{-1}
\end{aligned}
$$

once $M_{R}$ and $\alpha_{i}^{-1}\left(M_{R}\right)$ 's are given.

In principle we can calculate $\alpha_{i}^{-1}\left(M_{R}\right)$ 's from low-energy experimental values of $\alpha_{i}^{-1}$ 's according to the RGE. We choose, however, another way to calculate $\alpha_{i}^{-1}\left(M_{R}\right)$ in order to avoid ambiguities such as SUSY breaking scale $M_{S}$, strong coupling $\alpha_{C}$ and so on. Because we already know the unification scale $M_{U}^{\mathrm{MSSM}}$ and $\alpha^{\mathrm{MSSM}_{U}^{-1}}\left(M_{U}\right)$ in MSSM GUT and above the intermediate scale considered in this paper all couplings $\alpha_{i}$ 's are small enough for one-loop approximation of RGE to work well, we calculate $\alpha_{i}^{-1}\left(M_{R}\right)$ 's from the input parameter $\alpha_{U}^{\mathrm{MSSM}_{U}^{-1}}\left(M_{U}\right)$ at the GUT scale $M_{U}^{\mathrm{MSSM}}$. We choose input parameters from ref.[6] as follows,

$$
M_{U}^{\mathrm{MSSM}}=10^{16.3} \mathrm{GeV}, \quad \alpha_{U}^{\mathrm{MSSM}_{U}^{-1}}\left(M_{U}\right)=25.7 .
$$

Then we select the matter contents which satisfy the criteria. A search was made for all possible combinations of matter contents. The reason why this is possible is that the number of each matter multiplet which we can take into account simultaneously is limited. If the number of a representation is sufficiently large, its contribution to beta functions is too big to keep the couplings $\alpha_{i}$ 's in the perturbative region below the unification scale $M_{U}$ (see constraint 2).

Now we present our results. The case II. is the most preferable group for us accepting it as the intermediate one. In this case, though many combinations of matter multiplets satisfy the conditions which we imposed, most combinations lead the gauge coupling $\alpha_{U}$ to the value of about $1 / 5$, which is beyond the acceptable region to apply the perturbation theory. 
There are only two combinations which result in small value of $\alpha_{U} \simeq 1 / 20$.

$$
\text { Solution (i) } \quad \alpha_{U}^{-1}\left(M_{U}\right)=19.0049, \quad M_{U}=10^{16.3} \mathrm{GeV}
$$

Higgs contents

$$
\begin{array}{llll}
(1,3,1)(6) & 1 & (1,3,1)(-6) & 1 \\
(2,2,1)(0) & 2 & & \\
(3,1,1)(0) & 1 & & \\
(1,1,8)(0) & 1 & &
\end{array}
$$

Solution (ii)

$$
\alpha_{U}^{-1}\left(M_{U}\right)=19.0049, \quad M_{U}=10^{16.3} \mathrm{GeV}
$$

Higgs contents

$$
\begin{array}{llll}
(1,3,1)(6) & 1 & (1,3,1)(-6) & 1 \\
(2,2,1)(0) & 1 & & \\
(2,1,1)(3) & 1 & (2,1,1)(-3) & 1 \\
(3,1,1)(0) & 1 & & \\
(1,1,8)(0) & 1 & &
\end{array}
$$

with an input parameter $M_{R}=10^{12} \mathrm{GeV}$. In case of another $M_{R}, \alpha_{U}^{-1}\left(M_{U}\right)$ is slightly varied, though $M_{U}$ does not change. In this table, for example, (1,3,1)(6) 1 stands for that the representation of the Higgs under $G_{2231}$ is $(1,3,1)(6)$ and its number is one. In the solution (i), $(1,3,1)( \pm 6)$ are contained in $\mathbf{1 2 6}$ of $S O(10)$ and possibly give Majorana mass to right-handed neutrino. $(2,2,1)(0)$ can be regarded as the standard Higgs in MSSM and belonging to $\mathbf{1 0}$ or $\mathbf{1 2 6}$ of $S O(10)$. $(3,1,1)(0)$ and $(1,1,8)(0)$ are involved in $\mathbf{4 5}$ of $S O(10)$ [10]. The unification of the gauge couplings of the solution (i) is represented in Figure 11.

In contrast to the fact that many viable combinations exist in the case II, there is no solution for the breaking chain of I, in which $S O(10)$ breaks down to SM through so-called Pati-Salam symmetry [1]. Therefore if we consider both supersymmetric GUT and the scenario described above, in which right-handed neutrino masses are given by the symmetry breaking at the intermediate scale, it is impossible to use the group $G_{224}$ as the intermediate group. In the breaking chain of III, there is no solution which passes all of the constraints except the case of $M_{R}=10^{14} \mathrm{GeV}$. which is a rather high scale as an intermediate scale. Moreover in this case $\alpha_{U}$ becomes larger than 0.1 at the GUT scale and we can not trust perturbative approach to the unified scale. So it is difficult for the group $G_{214}$ to be realized at high energy region in this scenario.

\footnotetext{
${ }^{3}$ We adopt the normalization for $U(1)_{B-L} T_{4}^{15}=\operatorname{diag}(1,1,1,-3)$.

${ }^{4}$ If ignoring the constraint 4 , there are solutions in the case that $M_{R} \geq 10^{12} \mathrm{GeV}$. Proton decay via colored Higgs is quite dependent on a model.
} 
Finally we give a summary. When we construct SUSY SO(10) GUT model under the assumption that the right-handed neutrinos acquire their masses of the intermediate scale by the renormalizable coupling (Yukawa coupling) and there is only one intermediate scale, among three cases $G_{2231}$ is the most favorable group to be built in. The reason why neither $G_{224}$ nor $G_{214}$ can be used is as follows. In case I, to give right-handed neutrinos masses, $(1,3,10)$ Higgs is needed. This representation, however, makes too large a contribution to beta functions to achieve a small coupling.

We thank T. Kugo, K. Inoue and Izawa, K-I. for useful discussions related to this work. M.B. is supported in part by Grant-in-Aid for Scientific Research from Ministry of Education, Science, and Culture(\#06640416). T.T. is JSPS Research Fellow and is supported in part by Grant-in-Aid for Scientific Research from Ministry of Education, Science, and Culture(\#3116).

Figure 1: Evolution of coupling constants under the breaking chain given in II and the Higgs contents in solution (i). $M_{R}$ is taken to be $10^{12} \mathrm{GeV}$. The calculation is based on one-loop approximation. 


\section{References}

[1] For a review, M. Fukugita and T. Yanagida, preprint YITP/K-1050.

[2] L. Wolfenstein, Phys. Rev. D17 (1978) 2369; S. P. Mikheev and A. Yu. Smirnov, Sov. J. Nucl. Phys. 42 (1985) 913

[3] T. Yanagida, in Proceedings of the Workshop on Unified Theory and Baryon Number in the Universe, ed. A. Sawada and H. Sugawara, (KEK, Thukuba, Japan, 1979); M. Gell-Mann, P. Ramond and R. Slansky, in Supergravity, ed. F. van Nieuwenhuizen and D. Freedman, (North Holland, 1979)

[4] E. Witten, Phys. Lett. 91B (1980) 81

[5] H. Georgi, in Particle and Fields, ed. C. E. Carlson, (A. I. P., 1975); H. Fritzsch and P. Minkowski, Ann.Phys 93 (1975) 193

[6] U. Amaldi, W.Boer and H. Fürstenau, Phys. Lett. B260 (1991) 447; P. Langacker and M. Luo, Phys. Rev. D44 (1991) 817

[7] M. T. Grisaru, M. Roček and W. Siegel, Nucl. Phys. B159 (1979) 429

[8] Y. Kawamura, H. Murayama and M. Yamaguchi, Phys. Lett. B324 (1994) 52; N.G. Deshpande, E. Keith and T.G. Rizzo, Phys. Rev. Lett. 70 (1993) 3189

[9] For a review, W. Lucha, Comm. Nucl. Part. Phys. 16 (1986) 155; P. Langacker, Phys. Rep. 72 (1981) 185

[10] R. Slansky, Phys. Rep. 79 (1981) 1

[11] J. C. Pati and A. Salam, Phys. Rev. D10 (1974) 275 